\title{
A CLASS OF CHARACTERISTIC-VALUE PROBLEMS
}

\author{
BY \\ R. M. MORONEY(1)
}

\section{Chapter I}

0. Introduction. The question of interest here is that of the existence of real numbers $\lambda_{i}$ such that a solution $x(\lambda, t)$ on $0 \leqq t \leqq 1$ of

$$
x^{\prime \prime}+x F(x, \lambda, t)=0,
$$

satisfying the initial conditions

$$
x(\lambda, 0)=0, \quad x^{\prime}(\lambda, 0)=1,
$$

also satisfies, when $\lambda=\lambda_{i}$, the end condition

$$
x\left(\lambda_{i}, 1\right)=0 .
$$

Throughout this paper all variables are supposed to be real and all functions real-single-valued.

The above problem has been treated by Nehari [1] for the case where $F(x, \lambda, t)=G\left(\lambda^{2} x^{2}, t\right)$. By taking $F(x, \lambda, t)=\lambda+q(t)$ the classical Sturm-Liouville problem results.

The principal results to be given are Theorems 1 and 2 of $\S 2$. These theorems give hypotheses on $F(x, \lambda, t)$ which assure the existence of infinitely many characteristic numbers $\lambda_{i}$, and which are "sharp" in a certain sense.

In $\$ 3$ it is shown that the theorems proved in $\$ 2$ apply to the Nehari and Sturm-Liouville problems and lead to an extension of known results.

It will be shown that (2) and (3) can be replaced by general separated boundary conditions.

Chapter II contains additional results which are summarized in a separate introductory section there.

1. Preliminaries. 1.1. Throughout this section $F(x, \lambda, t)$ is assumed to be a function defined on the set $\{(x, \lambda, t):-\infty<x<\infty, \alpha \leqq \lambda<\infty, t \in I\}$ for some number $\alpha$ and some closed and bounded interval $I$, and to be continuous in $(x, \lambda, t)$ there. In addition $F$ is supposed to be positive (except possibly where $x=0$ ) and to satisfy for fixed $\lambda$ and $t$

$$
x_{2}>x_{1} \geqq 0 \text { or } \quad x_{2}<x_{1} \leqq 0 \Rightarrow F\left(x_{2}, \lambda, t\right) \geqq F\left(x_{1}, \lambda, t\right) .
$$

Satisfaction of this last condition will be called the monotonicity property of $F$.

Received by the editors April 27, 1961.

(1) The author is indebted to Professor N. Levinson for suggesting the research reported on here. This paper is extracted from a doctoral dissertation presented at the Massachusetts Institute of Technology. The inspiration and assistance of Professor Gian-Carlo Rota, who supervised the thesis, are gratefully acknowledged. 
The object of this preliminary section is to define a crucial property which $F$ must possess, which will be called the regenerative property. This definition is made in paragraph 1.6, using other definitions introduced in the interim.

1.2. Definition. Let $P$ represent the collection of all functions $f(s)$ which are defined and positive on some interval $[\rho, \infty)$ ( $\rho$ need not be the same for each member of $P$ ).

Definition. $G=\left\{f(s): f \in P\right.$ and $\left.\lim _{s \rightarrow \infty} f(s)=0\right\}$.

\subsection{Definition.}

$$
\check{F}(x, \lambda)=\sup _{t \in I} F(x, \lambda, t), \quad \hat{F}(x, \lambda)=\inf _{t \in I} F(x, \lambda, t) .
$$

Clearly $\check{F}$ and $\hat{F}$ are defined, and positive unless $x=0$, on $\{(x, \lambda):-\infty<x<\infty, \alpha \leqq \lambda<\infty\}$. It is claimed in addition that $\check{F}$ and $\hat{F}$ are continuous in $(x, \lambda)$, and enjoy the monotonicity property, on the same $(x, \lambda)$ set as above.

Proof of continuity. The proof will be given for $\check{F}$; a similar argument is valid for $\hat{F}$. Suppose $\breve{F}(x, \lambda)$ fails to be continuous at $(\bar{x}, \bar{\lambda})$. This means that there exists an $\epsilon>0$ and a sequence of pairs $\left\{\left(x_{i}, \lambda_{i}\right)\right\}$ such that $\left(x_{i}, \lambda_{i}\right)$ $\rightarrow(\tilde{x}, \tilde{\lambda})$ but $\left|\check{F}(\tilde{x}, \tilde{\lambda})-\check{F}\left(x_{i}, \lambda_{i}\right)\right|>\epsilon$. For each $i$ let $t_{i}$ be any value of $t$ such that $F\left(x_{i}, \lambda_{i}, t_{i}\right)=\check{F}\left(x_{i}, \lambda_{i}\right)$. By the compactness of $I$ there is at least one such $t_{i}$ for each $i$.

Since the $t_{i}$ are members of a compact set, there exists a convergent subsequence, say $t_{i k}$. Let $\lim t_{i k}=\tilde{t}$. We have $\left(x_{i k}, \lambda_{i k}, t_{i k}\right) \rightarrow(\tilde{x}, \tilde{\lambda}, \tilde{t})$, so by the continuity of $F, F\left(x_{i k}, \lambda_{i k}, t_{i k}\right) \rightarrow F(\tilde{x}, \tilde{\lambda}, \tilde{t})$. Because $F\left(x_{i k}, \lambda_{i k}, t_{i k}\right)=\check{F}\left(x_{i k}, \lambda_{i k}\right)$ however, the inequality of the preceding paragraph must apply in the limit, i.e. $\check{F}(\tilde{x}, \tilde{\lambda})-F(\tilde{x}, \tilde{\lambda}, \tilde{t}) \geqq \epsilon$, where the magnitude sign has been removed because of the definition of $F$.

Let $\check{t}$ be such that $\check{F}(\bar{x}, \tilde{\lambda})=F(\bar{x}, \tilde{\lambda}, \tilde{t})$. By continuity and the foregoing, $F\left(x_{i k}, \lambda_{i k}, \check{l}\right)>F\left(x_{i k}, \lambda_{i k}, t_{i k}\right)$ for large enough $k$. This is a contradiction of the choice of $t_{i k}$.

Proof of monotonicity. Again the proof is given only for $\breve{F}:$ if $x_{2}>x_{1} \geqq 0$ or $x_{2}<x_{1} \leqq 0$ there will exist $t_{2}$ and $t_{1}$ such that

$$
\check{F}\left(x_{2}, \lambda\right)=F\left(x_{2}, \lambda, t_{2}\right) \geqq F\left(x_{2}, \lambda, t_{1}\right) \geqq F\left(x_{1}, \lambda, t_{1}\right)=\check{F}\left(x_{1}, \lambda\right) .
$$

1.4. Clearly the following transformations are defined on $P$ and map $P$ into itself :

$$
\begin{aligned}
T^{+}:\left(T^{+} f\right)(s) & =f(s) \check{F}(f(s), s), \\
T^{-}:\left(T^{-} f\right)(s) & =f(s) \check{F}(-f(s), s), \\
U^{+}:\left(U^{+} f\right)(s) & =\hat{F}(f(s), s), \\
U^{-}:\left(U^{-} f\right)(s) & =\hat{F}(-f(s), s) .
\end{aligned}
$$


1.5. If $f(s) \in G$ as defined above, $\lim _{s \rightarrow \infty} U^{+} f$ may exist and be infinite, exist and be finite, or fail to exist. Exactly the same possibilities arise for $\lim _{s \rightarrow \infty} U^{-} f$. If the first alternative holds for both transforms we shall write $\lim _{s \rightarrow \infty} U^{ \pm} f=\infty$. This makes precise the Definition $H=\{f(s): f \in G$ and $\left.\lim _{s \rightarrow \infty} U^{ \pm} f \neq \infty\right\}$.

1.6. Definition. $F(x, \lambda, t)$ has the regenerative property iff

(a) For each fixed $x \neq 0, \lim _{\lambda \rightarrow \infty} \hat{F}(x, \lambda)=\infty$.

(b) $T^{+} H \subset H, T-H \subset H$.

It is to be observed that this definition is meaningful regardless of whether $F$ has the monotonicity property. The reason for the name regenerative will be seen in the proof of Lemma 5. The following two examples will be useful in $\S 3$ :

Example 1 . On the set $\{(x, \lambda, t):-\infty<x<\infty, \lambda \geqq 0,0 \leqq t \leqq 1\}$ the function $[\lambda+q(x, \lambda, t)]$ is regenerative if $q$ is bounded.

Proof. The first condition, that is (a) of paragraph 1.6, is clear and the second follows from the fact that the set $H$ is empty.

EXAmple 2. On the same set of $(x, \lambda, t)$ as in Example 1 the function $Q\left(\lambda^{2} x^{2}, t\right)$ is regenerative if $Q(u, v)$ satisfies the following conditions:

(i) $Q$ is defined and continuous in $(u, v)$ on $\{(u, v): u \geqq 0,0 \leqq v \leqq 1\}$.

(ii) $\lim _{u \rightarrow \infty} Q(u, v)=\infty$ uniformly in $v$.

Proof. Condition (a) follows from (ii). Since $Q\left(\lambda^{2} x^{2}, t\right)$ is even in $x, T^{+}$ $=T^{-}=T, U^{+}=U^{-}=U$, and the continuity of $Q$, along with condition (ii), assure that

$$
\begin{aligned}
u \grave{Q}\left(u^{2}\right) & \rightarrow \infty \Leftrightarrow u \rightarrow \infty, \\
\hat{Q}(u) & \rightarrow \infty \Leftrightarrow u \rightarrow \infty .
\end{aligned}
$$

Thus $f(s)$ in $H \Rightarrow \hat{Q}\left(s^{2} f^{2}(s)\right) \rightarrow \infty \Rightarrow s f(s) \rightarrow \infty \Rightarrow s f(s) \check{Q}\left(s^{2} f^{2}(s)\right) \rightarrow \infty \Rightarrow \hat{Q}(s f(s) \varnothing)$ $\rightarrow \infty$, i.e. $T f \in H$ and condition (b) is satisfied.

The next two examples show that conditions (a) and (b) in the definition of the regenerative property are independent, even when $F$ possesses the monotonicity property. (The domain in each case is the same as in Example 1.)

EXAMPLE 3. $(\lambda+1)^{-1}$ is not regenerative.

Proof. Here $H=G$, so (b) holds but (a) fails.

EXAMPLE 4. $\lambda\left(x^{2}\right)^{(3+t) / 4}$ is not regenerative.

Proof. If $g(s)$ is a member of $G$ then, by the definition of $G, g(s)$ is eventually less than 1, hence for sufficiently large $s \hat{F}(g(s), s)=\inf s\left[g^{2}(s)\right]^{(3+t) / 4}$ $=s g^{2}(s)$ and, by a similar derivation, $\breve{F}(g(s), s)=s\left[g^{2}(s)\right]^{3 / 4}$. Thus $s^{-1 / 2}$ is a member of $H$ (since $U\left(s^{-1 / 2}\right)=1$ ), but $T\left(s^{-1 / 2}\right)=s^{-1 / 2}\left(s s^{-3 / 4}\right)=s^{-1 / 4}$ which is not a member of $H ; U\left(s^{-1 / 4}\right)=s^{1 / 2} \rightarrow \infty$. Condition (b) of paragraph 1.6 does not hold, whereas (a) clearly does. Both this function and the one in the preceding example have the monotonicity property. 
2. Results. With the preceding definitions it is possible to give a concise statement of our first main result.

THEOREM 1. Let $D$ be the set $\{(x, \lambda, t):-\infty<x<\infty, \alpha \leqq \lambda<\infty, 0 \leqq t \leqq 1\}$. Let $F(x, \lambda, t)$ be defined on $D$ and satisfy the following hypotheses:

(i) $F$ is continuous in $(x, \lambda, t)$ on $D$.

(ii) For each fixed $\lambda$ and positive $N$ there exists a $K(\lambda, N)$ such that whenever $\left|x_{2}\right| \leqq N$ and $\left|x_{1}\right| \leqq N,\left|F\left(x_{2}, \lambda, t\right)-F\left(x_{1}, \lambda, t\right)\right| \leqq K(\lambda, N)\left|x_{2}-x_{1}\right|$ uniformly in $t$.

(iii) There exists $a \rho \geqq \alpha$ such that, for $\lambda \geqq \rho$ and $(x, \lambda, t)$ in $D, F(x, \lambda, t) \geqq 0$ and $F=0$ only when $x=0$. For each $\lambda \in[\alpha, \rho]$ there exists a solution of $(1)+(2)$ on $[0,1]$.

(iv) For fixed $\lambda$ and $t, \lambda \geqq \rho, x_{2}>x_{1} \geqq 0$ or $x_{2}<x_{1} \leqq 0 \Rightarrow F\left(x_{2}, \lambda, t\right) \geqq F\left(x_{1}, \lambda, t\right)$, i.e. $F$ has the monotonicity property for large $\lambda$.

(v) $F$ has the regenerative property. Then

(a) For each $\lambda$ in $[\alpha, \infty)$ there exists a unique solution of (1) and the initial conditions (2) on $0 \leqq t \leqq 1$.

(b) If $M$ is defined as the number of zeros in $(0,1)$ of the solution as in (a) for $\lambda=\alpha$, there exists an infinite sequence of characteristic numbers $\alpha \leqq \lambda_{M}<\lambda_{M+1}$ $<\cdots<\lambda_{M+n}<\cdots$ such that the solution as in (a) for $\lambda=\lambda_{i}$ also satisfies the end conditions (3). Furthermore, this solution has precisely $i$ zeros in $(0,1)$.

The proof of this theorem involves a sequence of lemmas. The first of these validates the existence and uniqueness assertions, while providing some additional facts which will be needed later.

Lemma 1. Let $F(x, \lambda, t)$ be defined for $t>1$ by $F(x, \lambda, t)=F(x, \lambda, 1)$. Then for this extended $F$, and every $\lambda$ in $[\rho, \infty)$, there exists a unique solution of $(1)+(2)$ on $[0, \infty)$. Furthermore, this solution has infinitely many zeros on $(0, \infty)$.

Proof of existence and uniqueness. Local existence is assured by hypothesis (i). Hypothesis (iii) and the form of (1) show that $x(t)$ and $x^{\prime \prime}(t)$ differ in sign, that is a solution curve is concave toward the $t$ axis as far as it exists. Thus if $x$ and $x^{\prime}$ are finite at any $t_{0}$ the solution may be continued to the right of $t_{0}$ and will remain finite. Since $F(x, \lambda, t)$ is defined for all finite $x$, it follows that a solution may be continued indefinitely. Hypothesis (ii) gives uniqueness, as is well known (and may be replaced by any other condition which does so-the only use of hypothesis (ii) is to assure uniqueness).

Proof of the infinity of zeros. Let $x(\lambda, t)$ be the solution of (1) $+(2)$ for some $\lambda$. It is claimed first that this solution has a zero for at least one $t \geqq 1$. If $x(\lambda, 1)=0$ this is true. If $x(\lambda, 1) \neq 0$ and $x x^{\prime}(\lambda, 1)<0$ the solution must lie between its tangent line and the $t$ axis, and hence is trapped, by the previously mentioned concavity of solution curves. There remains then only the 
case where $x(\lambda, 1) \neq 0$ and $x x^{\prime}(\lambda, 1) \geqq 0$. In this case, since $F(x, \lambda, t)$ is nondecreasing in $x$ and independent of $t$ on $t \geqq 1$, the magnitude of $x^{\prime \prime}$ can not decrease while $|x(\lambda, t)| \geqq|x(\lambda, 1)|$, and is nonzero at $t=1$, so ultimately $x^{\prime}$ must change sign. The argument of the preceding case then applies.

Once it is seen that $x(\lambda, t)$ has at least one zero to the right of $t=1$ it is easy to see that it has infinitely many. Indeed at such a zero $x^{\prime} \neq 0$ (by the uniqueness of the identically zero solution) and the arguments of the preceding section may be applied. I

The next four lemmas lead to an expression of the way in which $F(x, \lambda, t)$ depends on $\lambda$. Notice that this is the function of hypothesis (v)-indeed $F(x, \lambda, t)$ could be independent of $\lambda$ and still satisfy all the other hypotheses of Theorem 1. What is needed here, however, is a description of this dependence of $F$ on $\lambda$ which is in a more appropriate form than hypothesis ( $v$ ). Lemma 5 gives the new description.

Lemma 2. Let $T^{+}, T^{-}$, and $G$ be as in $\S 1$, with $F$ the $F$ of Theorem 1. There exist transformations $R^{+}, R^{-}$, defined for all $g$ in $G$, such that for these $g$ :

(a) $R^{+} g \in G, R^{-} g \in G$.

(b) $T^{+}\left(R^{+} g\right)=g, T^{-}\left(R^{-} g\right)=g$.

Proof. The existence of $R^{+}$will be demonstrated. A similar proof may be used for $R^{-}$.

Given $g$ in $G$, construct $R^{+} g$ as follows: for each fixed sufficiently large $s$ solve

$$
x \check{F}(x, s)=g(s)
$$

for $x$. This is uniquely possible (for $s$ greater than some number) because the left side of (4) is zero at $x=0$ and strictly increasing to $\infty$ in $x$ (recall that $\check{F}(x, s)$ has the monotonicity property), while the right side is some positive number whenever $g$ is defined.

It must now be shown that $R^{+} g$ as constructed above satisfies (a) and (b). (b) is clear. As for (a), note that for fixed $x$ the right side of (4) tends to zero as $s$ tends to infinity $(g \in G)$, while the left side tends to infinity by property (a) of regenerative functions. Thus if $\left(R^{+} g\right)(s)$ does not tend to zero as $s$ tends to infinity the left side of (4) can not tend to zero, which is a contradiction.

Lemma 3. $G-H$ is nonempty.

Proof. For $0<x \leqq 1$ define a function $k(x)$ by $k(x)=\inf \{s: s \geqq \rho$ and $\hat{F}(x, y)$ $\geqq 1 / x$ for all $y \geqq s\}$. Condition (a) on regenerative functions (in paragraph 1.6) assures that the set $\{s: \hat{F}(x, y) \geqq 1 / x$ for all $y \geqq s\}$ is nonempty for each $x>0$, hence the set of which $k(x)$ is the inf is nonempty and has a lower bound $(\rho)$, so $k(x)$ is well-defined.

Since $\hat{F}(x, \rho)$ is bounded on $0 \leqq x \leqq 1, k(x)>\rho$ for sufficiently small $x$. Let 
$0<x \leqq \delta$ be an interval where this is true, and let $p(x)$ be the restriction of $k(x)$ to $(0, \delta]$. By the continuity of $\hat{F}, \hat{F}(x, p(x))=1 / x$. It will now be shown that $p(x)$ has the following additional properties:

(i) $p(x)$ is strictly decreasing in $x$.

(ii) $p(x) \rightarrow \infty$ as $x \rightarrow 0$.

(iii) $p$ is continuous.

Proof of (i). If $x_{2}>x_{1}$ and $t \geqq 0$ then $\hat{F}\left(x_{2}, p\left(x_{1}\right)+t\right) \geqq \hat{F}\left(x_{1}, p\left(x_{1}\right)+t\right) \geqq 1 / x_{1}$ (by the way in which $k(x)$ is defined) and thus, since $1 / x_{1}>1 / x_{2}, \hat{F}\left(x_{2}, p\left(x_{1}\right)+t\right)$ $>1 / x_{2}$. Since this is true for all non-negative $t, p\left(x_{1}\right)$ is a member of the set of which $p\left(x_{2}\right)$ is the inf, i.e. $p\left(x_{2}\right) \leqq p\left(x_{1}\right)$. Equality can not hold, however, because $\hat{F}\left(x_{2}, p\left(x_{2}\right)\right)=1 / x_{2}$.

Proof of (ii). If $p(x) \rightarrow \infty$ as $x \rightarrow 0$ there exists an $M$ such that $p(x) \leqq M$, since $p$ is decreasing by (i). But this implies $\hat{F}(x, M) \geqq 1 / x$ (again by the way in which $k$ is defined) for all $x>0$, and this is a contradiction since $\hat{F}(x, M)$ is bounded on $0 \leqq x \leqq 1$.

Proof of (iii). Since $p$ is decreasing it is enough to show that there is no $t$ in $(0, \delta)$ such that $p(t+)<p(t)$ and/or $p(t-)>p(t)$. Suppose first that for some $t$ in $(0, \delta), p(t+)<p(t)$. Then there exists a $\tau, p(t+)<\tau<p(t)$, such that $\hat{F}(t, \tau)<1 / t$, since otherwise $p(t)$ could be decreased. However, since $\tau>p(t+)$ $>p(t+\epsilon)$ for every $\epsilon>0, \hat{F}(t+\epsilon, \tau) \geqq 1 /(t+\epsilon)$. Letting $\epsilon \rightarrow 0$ gives a contradiction.

Next suppose that for some $t$ in $(0, \delta] p(t-)>p(t)$. Then there exists a $\tau, p(t-)>\tau>p(t)$, such that for every $\epsilon>0 F(t-\epsilon, \tau) \leqq 1 /(t-\epsilon)$ but $F(t, \tau)$ $>1 / t$. Again a contradiction of the continuity of $F$ is reached.

To recapitulate, it has been shown so far that on a sufficiently small $x$ interval $(0, \delta]$ there exists a continuous monotone-decreasing function $p(x)$ which tends to infinity as $x \rightarrow 0$ and is a solution of $\hat{F}(x, p(x))=1 / x$. Define a function $g(s)$ on the range of $p$ by $g(p(x))=x$. This is possible by the monotonicity of $p$.

For any sufficiently large $s$ there exists a $u$ such that $s=p(u)$, hence $g(s)$ is defined and $\hat{F}(g(s), s)=\hat{F}(g(p(u)), p(u))=\hat{F}(u, p(u))=1 / u$. As $s \rightarrow \infty$ the $u$ such that $s=p(u)$ tends to zero, that is, $\hat{F}(g(s), s) \rightarrow \infty$, and $g(s) \rightarrow 0$. Thus $g(s)$ is in $G$ and $\lim _{s \rightarrow \infty} U^{+} g=\infty$. A similar construction will yield an $h(s)$ in $G$ such that $\lim _{s \rightarrow \infty} U^{-} h=\infty$. Then $h(s) \vee g(s)$ will be the desired member of $G-H$.

Lemma 4. $R^{+}(G-H) \subset(G-H), R^{-}(G-H) \subset(G-H)$.

Proof. By Lemma $2 R^{+} G \subset G$. If, then, there existed an $h$ in $(G-H)$ such that $R^{+} h \notin(G-H)$ necessarily $R^{+} h \in H$. Then $T^{+}\left(R^{+} h\right)=h \in(G-H)$, contradicting the fact (second condition of regeneracy) that $T^{+} H \subset H$. Again, a similar proof for $R^{-}$. I

Lемма 5. For every positive integer $n$ there exist $(n+2)$ functions in $G: g(s)$, $C_{0}(s), C_{1}(s), \cdots, C_{n}(s)$ such that 
(a) For $j=0,1, \cdots, n$

$$
\lim _{s \rightarrow \infty} g^{3}(s) \hat{F}\left((-)^{i} C_{j}(s), s\right)=\infty
$$

(b) For sufficiently large s and $j=0,1, \cdots, n-1$

$$
20 g(s) C_{j+1}(s) \check{F}\left((-)^{j+1} C_{j+1}(s), s\right)<C_{j}(s) .
$$

Proof. Take for $C_{0}(s)$ any member of $G-H$. This is possible by Lemma 3 . Then take $C_{1}(s)=\left(R^{-} C_{0}\right)(s), \quad C_{2}=R^{+} C_{1}, \cdots, C_{2 m}=R^{+} C_{2 m-1}, \quad C_{2 m+1}$ $=R^{-} C_{2 m}, \cdots$. By Lemma 4 all the $C_{j}$ are in $G-H$ (this is why $F$ is said to be regenerative; starting with any function $h$ in $G-H$ one can find a sequence of transforms of this function, to wit $\left(R^{+}\right)^{n} h$ or $\left(R^{-}\right)^{n} h$, which are themselves in $G-H: F$ "generates" new members of $G-H)$.

By the construction, for $j$ even $R-C_{j}=C_{j+1}$ and

$$
T^{-}\left(R^{-} C_{j}\right)=C_{j}=T^{\sim} C_{j+1}=C_{j+1} \check{F}\left(-C_{j+1}(s), s\right),
$$

that is, reading the second and last terms, (b) is satisfied for any $g(s)$ in $G$. A similar calculation shows that (b) is also satisfied for $j$ odd. Since all the $C_{j}$ are in $G-H$, (a) will hold if $g(s)$ does not tend to zero too fast. For any fixed $j$

$$
g_{j}(s)=\left[\hat{F}\left((-)^{j} C_{j}(s), s\right)\right]^{-1 / 4}
$$

is a member of $G$ such that

$$
\lim _{s \rightarrow \infty} g_{j}^{3}(s) \hat{F}\left((-)^{j} C_{j}(s), s\right)=\infty,
$$

so a suitable choice of $g$ would be $\inf _{j} g_{j}(s)$.

REMARK. The statement of Lemma 5 may replace the hypothesis that $F$ is regenerative in Theorem 1, since the only use of that hypothesis in the proof, as will be seen, is to assure the existence of functions as in Lemma 5 .

Proof of Theorem 1. With the preceding lemmas established, the proof of Theorem 1 proceeds along lines similar to the Prüfer demonstration for the Sturm-Liouville problem.

For each $\lambda$ in $[\rho, \infty)$ let $x(\lambda, t)$ be the unique solution of $(1)+(2)$ whose existence is assured by Lemma 1. Put

$$
\theta(\lambda, t)=\int_{0}^{t} \frac{\left[x^{\prime}(\lambda, s)\right]^{2}+[x(\lambda, s)]^{2} F(x(\lambda, s), \lambda, s)}{\left[x^{\prime}(\lambda, s)\right]^{2}+[x(\lambda, s)]^{2}} d s .
$$

$\theta(\lambda, t)$ is well-defined because the uniqueness of the identically zero solution of (1) assures the nonvanishing of the denominator of the (continuous) integrand. It is easy to see that

$$
\theta(\lambda, t)=\tan ^{-1}\left[\frac{x(\lambda, t)}{x^{\prime}(\lambda, t)}\right]
$$


and

$$
\theta^{\prime}(\lambda, t)=\cos ^{2} \theta(\lambda, t)+\sin ^{2} \theta(\lambda, t) F(x(\lambda, t), \lambda, t) .
$$

Because of (8), $x(\lambda, 1)=0$ iff $\theta(\lambda, 1)=n \pi$ for some integer $n$. Recall that $M$ was defined in the statement of Theorem 1 as the number of zeros in $(0,1)$ of the solution of $(1)+(2)$ for $\lambda=\alpha$. Thus, since $\theta(\alpha, t)$ is increasing in $t$ by (9),

$$
M \pi<\theta(\alpha, 1) \leqq(M+1) \pi .
$$

The uniqueness of the solution $x(\lambda, t)$ of $(1)+(2)$ assures that $x(\lambda, t)$ and $x^{\prime}(\lambda, t)$ are continuous in $\lambda$ uniformly on $0 \leqq t \leqq 1$ [2]. Thus by $(7) \theta(\lambda, 1)$ is continuous in $\lambda$, hence takes intermediate values, and Theorem 1 will be proved when it is shown that $\theta(\lambda, 1) \rightarrow \infty$ as $\lambda \rightarrow \infty$. So

Lemma 6. $\theta(\lambda, 1) \rightarrow \infty$ as $\lambda \rightarrow \infty$.

Proof. It will be shown that for $p$ an arbitrary positive integer there exists a number $u$ such that for all $\lambda \geqq u, \theta(\lambda, 1) \geqq p \pi$. To avoid the powers of $(-1)$ which are so cumbersome in Lemma 5 , it will be assumed that $F(x, \lambda, t)$ is even in $x$. The necessary modifications for the case where $F$ is not even will be obvious.

2.1. Let $p$ be given, and let $g(s), C_{0}(s), \cdots, C_{p}(s)$ be the set of $(p+2)$ functions whose existence is assured by Lemma 5 . Choose $u$, once and for all, such that for any $\lambda \geqq u$

(a) $g(\lambda), C_{0}(\lambda), \cdots, C_{p}(\lambda)$ are defined and $<1$.

(b) $g^{3}(\lambda) \hat{F}\left(C_{j}(\lambda), \lambda\right)>\pi, j=1,2, \cdots, p$.

(c) $(5 p) g(\lambda)<1$.

(d) For $j=0,1, \cdots, p-1$,

$$
C_{j}(\lambda)>20 g(\lambda) C_{j+1}(\lambda) \check{F}\left(C_{j+1}(\lambda), \lambda\right) .
$$

(e) $C_{j+1}(\lambda)<1 / 2 C_{j}(\lambda), j=0,1, \cdots, p-1$.

That such a choice of $u$ is possible follows by Lemma 5: (b) and (d) above directly from (a) and (b) of Lemma 5, (a) and (c) above from the fact that all the functions are in $G$, (e) from (b) and (d).

Throughout the following arguments $\lambda$ is supposed to have some (any) fixed value greater than $u$, and will not be written, i.e. $g=g(\lambda), C_{n}=C_{n}(\lambda)$, $\theta(t)=\theta(t, \lambda)$ etc.

2.2. Since $\theta(0)=0$ by (7), and $\theta(t) \rightarrow \infty$ as $t \rightarrow \infty$ by Lemma 1 , there is an $s>0$ such that $\sin \theta(s)=g$ and $0 \leqq \sin \theta(t) \leqq g$ for $0 \leqq t \leqq s$. From (9), on $0<t$ $<s(\sin \theta)^{\prime}(t)=\cos \theta(t) \theta^{\prime}(t) \geqq \cos ^{3} \theta(t)$ (by using only the first term of (9)), and this in turn is greater than $\left[1-g^{2}\right]^{3 / 2}$. For some $\xi$ in $(0, s)$

$$
g=\sin \theta(s)-\sin \theta(0)=s\left[(\sin \theta)^{\prime}(\xi)\right]
$$

or, solving for $s$ 


$$
s=\frac{g}{(\sin \theta)^{\prime}(\xi)}
$$

Finally, using (c) of paragraph 2.1 and the hold on $(\sin \theta)^{\prime}$,

$$
s \leqq \frac{g}{\left[1-g^{2}\right]^{3 / 2}}<2 g .
$$

2.3. It is claimed that $x(s)>C_{1}$. Suppose the contrary. Then since

$$
\frac{x(s)}{x^{\prime}(s)}=\tan \theta(s)>\sin \theta(s)=g
$$

there follows $x^{\prime}(s)<C_{1} / g$. Clearly $x(t)$ is increasing on $0 \leqq t \leqq s$, hence on that interval

$$
\left|x^{\prime \prime}(t)\right|=x(t) F(x(t), u, t) \leqq C_{1} \check{F}\left(C_{1}(u), u\right)
$$

and

$$
\begin{aligned}
1 & =x^{\prime}(0)=\left[x^{\prime}(0)-x^{\prime}(s)\right]+x^{\prime}(s) \leqq s \sup \left|x^{\prime \prime}(t)\right|+\frac{C_{1}}{g} \\
& \leqq 2 g C_{1} \check{F}\left(C_{1}(u), u\right)+\frac{C_{1}}{g} .
\end{aligned}
$$

This is a contradiction, however, because by (b) of paragraph 2.1

$$
\begin{aligned}
\frac{2 g C_{1} \check{F}\left(C_{1}(u), u\right)}{C_{1} / g} & =2 g^{2} \check{F}\left(C_{1}(u), u\right) \\
& >g^{3} \check{F}\left(C_{1}(u), u\right)>\pi
\end{aligned}
$$

or, using (d) and (a) of paragraph 2.1

$$
2 g C_{1} \check{F}\left(C_{1}(u), u\right)+C_{1} / g<4 g C_{1} \check{F}\left(C_{1}\right)<C_{0}<1 .
$$

2.4. Next, follow $x(t)$ until $\sin \theta$ again reaches $g$, say at $t=v$. This is possible because as $t$ runs from 0 to $t_{1}$-the first zero of $x(t)$ (which exists by Lemma 1) $-\sin \theta$ increases from 0 to 1 and then decreases to 0 .

Clearly $x^{\prime}(v)<0$. It is claimed that $x(v)>C_{p}$. Suppose the contrary, and let $\check{t}$ be the point in $\left[0, t_{1}\right]$ at which $x(t)$ attains its maximum. Because $x(\check{t})$ $>x(s) \geqq C_{1}>C_{p}$, a point $w$ can be found, $\check{t}<w<v$, such that $x(w)=C_{p}$, $x^{\prime}(w)<0$, and $\sin \theta(w)>g$. As in paragraph 2.3, $\left|x^{\prime}(w)\right|<C_{p} / g$. Since for $s \leqq t \leqq w \sin \theta(t)>g$ and $x(t) \geqq C_{p}$,

$$
\pi>\theta(w)-\theta(s)=(w-s) \theta^{\prime}(\xi) \geqq(w-s) g^{2} \hat{F}\left(C_{p}(u), u\right)
$$

or, using (b) of paragraph 2.1, 


$$
w-s \leqq \frac{\pi}{g^{2} \hat{F}\left(C_{p}(u), u\right)}<g .
$$

By the concavity of the graph of $x(t)$ and the above

$$
x(\check{t}) \leqq(w-\check{t})\left|x^{\prime}(w)\right|+x(w)<2 C_{p}<C_{1}
$$

which is a contradiction.

2.5. Since $x(v) \geqq C_{p}$, the same argument as in paragraph 2.4 shows that $(v-s)<$ g. An argument as in paragraph 2.2 then gives $\left(t_{1}-v\right)<2 g$. Thus it has been shown that $t_{1}$-the first zero of $x(t)$-is such that $t_{1}<5 g$. The concavity of the graph of $x(t)$ assures that

$$
\left(t_{1}-\check{t}\right)\left|x^{\prime}\left(t_{1}\right)\right|>x(\grave{t})
$$

or

$$
\left|x^{\prime}\left(t_{1}\right)\right|>\frac{x(\check{t})}{t_{1}-\check{t}}>\frac{C_{1}}{t_{1}}>\frac{C_{1}}{5 g} .
$$

2.6. Let $t_{j}$ be the $j$ th zero of $x(t)$ and assume inductively that it has been shown for $j=1,2, \cdots, n<p$ that

(a) $t_{j}-t_{j-1}<5 g$,

(b) $\left|x^{\prime}\left(t_{j}\right)\right|>C_{j} / 5 g$.

Then by arguments similar to those already given, the same conclusions are established for $j=n+1$, viz.:

2.2. There exists a smallest $s>t_{n}$ such that

$$
|\sin \theta(s)|=g, \text { and }\left(s-t_{n}\right)<2 g \text {. }
$$

2.3'. $|x(s)|>C_{n+1}$, since otherwise

$$
C_{n} / 5 g<\left|x^{\prime}\left(t_{n}\right)\right| \leqq 2 g C_{n+1} \check{F}\left(C_{n+1}(u), u\right)+\frac{C_{n+1}}{g}
$$

and

$$
2 g C_{n+1} \check{F}\left(C_{n+1}(u), u\right)+C_{n+1} / g<4 g C_{n+1} \check{F}\left(C_{n+1}(u), u\right),
$$

leading to the contradiction (of (d) in step 2.1)

$$
C_{n}<20 g^{2} C_{n+1} \check{F}\left(C_{n+1}(u), u\right) .
$$

2.4'. Replace $C_{1}$ by $C_{n+1}$.

2.5'. Replace $C_{1}$ by $C_{n+1}$.

Thus by induction (a) and (b) hold for $j=1,2, \cdots, p$ and in particular

$$
t_{p}=\left(\left[t_{p}-t_{p-1}\right]+\left[t_{p-1}-t_{p-2}\right]+\cdots+\left[t_{1}-t_{0}\right]\right) \leqq 5 p g<1 .
$$

As already noted, this completes the proof of Theorem 1. 
In the next theorem the end conditions (2) and (3) are replaced by the more general conditions

$$
\begin{gathered}
x(0)=a, \quad x^{\prime}(0)=b, \quad\left(a^{2}+b^{2}\right) \neq 0 ; \\
c x(1)+d x^{\prime}(1)=0, \quad\left(c^{2}+d^{2}\right) \neq 0 .
\end{gathered}
$$

In addition, the statement of the theorem incorporates certain hypotheses weaker than those of Theorem 1 . The next definition makes precise the statement " $\theta$ takes intermediate values."

Definition. Let $x_{1}\left(\lambda_{1}, t\right)$ be some solution of (1) $+(13)$ for $\lambda=\lambda_{1}$, which exists on $[0,1]$ and does not vanish together with its derivative at any point of $[0,1]$, so that $\theta_{1}\left(\lambda_{1}, t\right)$ is well-defined by $(7)$. Let $x_{2}\left(\lambda_{2}, t\right)$ and $\theta_{2}\left(\lambda_{2}, t\right)$ be a similar solution and its argument function for $\lambda=\lambda_{2}$. The statement $\theta$ takes intermediate values means that for any two solutions as above, and any number $r$ in the interval $\left(\theta_{1}\left(\lambda_{1}, 1\right), \theta_{2}\left(\lambda_{2}, 1\right)\right)$, there exists a $\lambda_{r}$ in $[\alpha, \infty)$ and a solution $x_{r}\left(\lambda_{r}, t\right)$ of $(1)+(13)$ such that $\theta_{r}\left(\lambda_{r}, t\right)$ exists and $\theta_{r}\left(\lambda_{r}, 1\right)=r$.

Theorem 2. Let $D$ be the set $\{(x, \lambda, t):-\infty<x<\infty, \alpha \leqq \lambda<\infty, 0 \leqq t \leqq 1\}$ for some $\alpha$. Let $F(x, \lambda, t)$ be defined on $D$ and satisfy there the following hypotheses:

(i) $F(x, \lambda, t)$ is continuous in $(x, \lambda, t)$.

(ii) There exists $a \rho \geqq \alpha$ such that for $\lambda \geqq \rho$ and $(x, \lambda, t)$ in $D$

$$
F(x, \lambda, t) \geqq 0 \text { and } F=0 \Rightarrow x=0 .
$$

(iii) For fixed $\lambda$ and $t, \lambda \geqq \rho$,

$$
x_{2}>x_{1} \geqq 0 \text { or } x_{2}<x_{1} \leqq 0 \Rightarrow F\left(x_{2}, \lambda, t\right) \geqq F\left(x_{1}, \lambda, t\right) .
$$

(iv) For every positive integer $n$ there exists a set of $(n+2)$ positive functions $g(s), C_{0}(s), C_{1}(s), \cdots, C_{n}(s)$ which are defined for all sufficiently large $s$, tend to zero as $s \rightarrow \infty$, and are such that for $k=1$ or $k=0$

(a) $\lim _{s \rightarrow \infty} g^{3}(s) \hat{F}\left((-)^{j+k} C_{j}(s), s\right)=\infty$.

(b) For sufficiently large $s$

$$
C_{j}(s)>20 g(s) C_{j+1} \check{F}\left((-)^{j+k+1} C_{j+1}\right) .
$$

Then if $\theta$ takes intermediate values, and $M$ is the least number of zeros in $(0,1)$ of any solution of (1) $+(13)$ for $\lambda=\alpha$, there exists an infinite sequence of numbers $\alpha<\lambda_{M+1}<\lambda_{M+2}<\cdots<\lambda_{M+n}<\cdots$ such that for $\lambda=\lambda_{j}$ there is a solution of $(1)+(13)$ which satisfies (14). This solution vanishes precisely $j$ times in $(0,1)$.

REMARK. The existence of a solution having $M$ zeros can not be asserted, as is shown by the example

$$
\begin{gathered}
x^{\prime \prime}+\lambda^{2} x=0, \quad \lambda \geqq 3 \pi / 4, \\
x(0)=0, \quad x^{\prime}(0)=1, \quad x^{\prime}(1)=0 .
\end{gathered}
$$


Here the smallest characteristic number is $3 \pi / 2$, with characteristic function $(2 / 3 \pi) \sin (3 \pi / 2) t$. This function has a zero in $(0,1)$, whereas the solution of the differential equation plus initial conditions for $\lambda=3 \pi / 4$ (which is $\alpha$ in this case) is $(4 / 3 \pi) \sin (3 \pi / 4) t$ and has no zeros in $(0,1)$, i.e. $M=0$.

LEMMA 7. If $\lambda \geqq \rho$, any solution of (1) which vanishes together with its derivative at some point of $[0,1]$ is identically zero on $[0,1]$.

Proof. Let $t$ be the point in $[0,1]$ such that $x(t)=x^{\prime}(t)=0$. It will be shown that $x(s) \equiv 0$ for $t \leqq s \leqq 1$. A trivial modification of the argument (which is due to Nehari [1]) would then establish the result on the entire interval.

Let $t_{\omega}$ be the largest number $v$ such that $x(s)=x^{\prime}(s)=0$ on $[t, v]$, and assume $t_{\omega}<1$. There is no interval $\left(t_{\omega}, \xi\right)$ on which $x(s)>0$, because if there were, two applications of the mean-value theorem would yield the existence of a point in the same interval for which $x^{\prime \prime}>0$, contradicting the form of (1). It follows that $t_{\omega}$ is the limit of a descending sequence of zeros of $x(t): t_{1}$ $>t_{2}>\cdots>t_{n}>\cdots$. In the interval from $t_{\omega}$ to $t_{j}$

$$
x(t)=\int_{t_{\omega}}^{t_{i}} G(t, s) x(s) F(x(s), \lambda, s) d s,
$$

where

$$
G(t, s)= \begin{cases}\frac{t-t_{\omega}}{t_{j}-t_{\omega}}\left(t_{j}-s\right), & s>t, \\ \frac{t_{j}-t}{t_{j}-t_{\omega}}\left(s-t_{\omega}\right), & s<t .\end{cases}
$$

Letting $M_{j}$ be the maximum magnitude of $x(t)$ in $\left[t_{\omega}, t_{j}\right]$, and noting that $|G(t, s)| \leqq 1 / 4\left(t_{j}-t_{\omega}\right)$ gives

$$
\begin{aligned}
M_{j} & \leqq \frac{1}{4} \int_{t_{\omega}}^{t_{j}}\left(t_{j}-t_{\omega}\right) M_{j} F\left(M_{j}, \lambda, s\right) d s \\
1 & \leqq \frac{1}{4}\left[\int_{t_{\omega}}^{t_{j}} F\left(M_{1}, \lambda, s\right) d s\right]\left(t_{j}-t_{\omega}\right),
\end{aligned}
$$

which is a contradiction since $\left(t_{j}-t_{\omega}\right) \rightarrow 0$.

Proof of Theorem 2. It was noted in the proof of Theorem 1 that hypothesis (ii) of that theorem was used only to assure uniqueness of a solution of (1) satisfying given initial conditions. Uniqueness was used in only two ways: to assure that $\theta(\lambda, 1)$ would take intermediate values and to assure that for $\lambda \geqq \rho$ only the identically zero solution of (1) could vanish along with its derivative at any $t$. Thus by Lemma 7 and the assumption in Theorem 2 that $\theta$ takes intermediate values, Lemma 1 with the uniqueness assertion deleted holds in the present case, hence $\theta(\lambda, 1)$ exists for every solution of $(1)+(13)$. 
Clearly (14) prescribes $\theta(\lambda, 1)$ modulo $\pi$, and thus since $\theta$ takes intermediate values Theorem 2 will be proved when it is shown that $\theta(\lambda, 1)$ takes arbitrarily large values. This is easily done by slight modifications in the proof of Lemma 6 (Lemma 5 holds in the present case by hypothesis). Three cases are distinguished:

CASE 1. $a=0$ in (13). Here $x^{\prime}(0)=b$ instead of 1 . The presence of $k$ in hypothesis (iv) assures that $b$ may be assumed positive. By increasing the $u$ of Lemma 6 if necessary, $C_{0}(\lambda)$ can be made smaller than $b$ for all $\lambda \geqq u$. The contradiction established in (12) will then continue to hold and Lemma 6 will hold.

CASE 2. $b=0$ in (13). By increasing the $u$ of Lemma 6 if necessary, $C_{1}(\lambda)$ can be made smaller than $|a|$ for all $\lambda \geqq u$. Define $\theta(\lambda, 0)=\pi / 2$ for all $\lambda$ and enter the proof of Lemma 6 at step number 2.4.

CASE 3. $a \neq 0$ and $b \neq 0$ in (13). The idea in this case is to define $\theta(\lambda, 0)$ in such a way that the proof of Lemma 6 may be entered at the step where it is shown that when $\sin \theta$ is $g$ the magnitude of $x$ exceeds $C_{1}$ (or $C_{p}$, the choice being determined by the sign of $x x^{\prime}(0)$, i.e. $\left.a b\right)$. To this end, increase $u$ in Lemma 6 until for the given $a$ and $b \sin \left(\tan ^{-1} a / b\right)$ is greater than $g$ and $|a|>C_{1}$. Then define $\theta(\lambda, 0)$ to be in $(0, \pi)$ and equal to $\tan ^{-1} a / b$. The choice of $u$ has assured that $|\sin \theta|>g$.

For $t>0$, define $\theta(\lambda, t)$ by adding the chosen value of $\theta(\lambda, 0)$ to the right side of (7). This will not affect (8) or (9). Lemma 6 may now be entered at either the third or fourth step.

The foregoing completes the proof of Theorem 2.

REMARK. It may be questioned whether the change of hypotheses from Theorem 1 to Theorem 2 has resulted in a more general theorem (aside from the question of boundary conditions-clearly Theorem 1 can be extended to handle the general boundary conditions treated by Theorem 2).

Example 4 of $\$ 1$ shows that a more general theorem does result, since for the function $F(x, \lambda, t)=\lambda\left(x^{2}\right)^{(3+t) / 4}$, which is not regenerative, Lemma 5 holds. To see this, note that $\lambda^{p-1 / 2}$ is in $G-H$ for any $p$ such that $0 \leqq p<1 / 2$. Given $n$ a positive integer, take $p=(2 / 7)^{n+2}$ and define $C_{n}(\lambda)=\lambda^{p-1 / 2}$. Then take $C_{n-1}(\lambda)=\lambda^{q-1 / 2}$, where $q$ is the smallest number such that

$$
T C_{n}=C_{n}(\lambda) \lambda\left[C_{n}(\lambda)\right]^{3 / 2} \leqq C_{n-1}(\lambda)=\lambda^{q-1 / 2},
$$

that is

$$
\lambda^{p+1 / 2}\left[\lambda^{p-1 / 2}\right]^{z / 2} \leqq \lambda^{q-1 / 2},
$$

or

$$
\lambda^{1 / 4+5 p / 2} \leqq \lambda^{2}
$$

giving

$$
q<7 p / 2=(2 / 7)^{n+1} .
$$


Since $0<q<1 / 2 C_{n-1}(\lambda)$ is in $G-H$, and an easy induction gives the desired collection of $C_{j} . g(\lambda)$ may then be chosen as in the proof of Lemma 5.

A more detailed discussion of this lack of sharpness of the regenerative hypothesis is given in Chapter II.

3. Applications. In this section the results of $\$ 2$ are applied to Nehari's problem and the Sturm-Liouville problem. Additional results for these problems will be found in the next chapter.

Nehari's problem. The boundary-value problem

$$
\begin{gathered}
x^{\prime \prime}+x G\left(x^{2}, t\right)=0, \\
x(0)=0, \quad x(1)=0
\end{gathered}
$$

has been investigated by Nehari $[1 ; 3]$, who obtained the results corresponding to Theorems 1 and 2 under the hypothesis that $G(u, t)$ be defined on $\{u \geqq 0,0 \leqq t \leqq 1\}$ and possess there the following properties:

(i) $G(u, t)$ is continuous in $(u, t)$.

(ii) $G(u, t)>0$ for $u>0$ and all $t$.

(iii) There exists an $\epsilon>0$ such that for each fixed $t u^{-\iota} G(u, t)$ is strictly increasing in $u$.

To recast this problem in the form (1) $+(2)+(3)$, let $x(t)$ be any solution of (15) such that $x(0)=0$, and let $\lambda=x^{\prime}(0)$. Then $z(t)=\lambda^{-1} x(t)$ satisfies

$$
\begin{gathered}
z^{\prime \prime}+z G\left(\lambda^{2} z^{2}, t\right)=0, \\
z(0)=0, \quad z^{\prime}(0)=1
\end{gathered}
$$

and, if $x(1)=0$,

$$
z(\lambda, 1)=0 .
$$

Thus $z$ is a solution of $(1)+(2)+(3)$ for the special case $F(x, \lambda, t)=G\left(\lambda^{2} x^{2}, t\right)$. Conversely, if for some $\lambda$ there exists a solution $z(\lambda, t)$ of $(17)+(18)+(19)$ having $n$ zeros in $(0,1)$, then $x(t)=\lambda z(\lambda, t)$ is a solution of $(15)+(16)$ with $n$ zeros in $(0,1)$.

If $G(u, v)$ satisfies (i), (ii) and (iii) above, then $G\left(\lambda^{2} z^{2}, t\right)$ in (17) satisfies all the hypotheses of Theorem 1 except the uniqueness condition. To see this, note that condition (iii) assures that $G(u, t) \rightarrow \infty$ as $u \rightarrow \infty$ uniformly in $t$, hence by Example 2 of $\S 1 G$ is regenerative; clearly the other hypotheses are met.

Since condition (iii) and continuity assure that $G(0, t)=0, \alpha$ in Theorem 1 may be taken as zero, whence $M=0$ and Theorem 1 assures the existence of a solution of $(15)+(16)$ having $i$ zeros in $(0,1)$ for $i=0,1, \cdots$, provided that a solution of (15) is uniquely determined by its initial values.

Because of this last stipulation, Theorems 1 and 2 do not include Nehari's result (unless it can be shown that conditions (i), (ii) and (iii) either give uniqueness or assure that $\theta$ takes intermediate values). On the other hand, 
Theorem 1 is applicable with a condition less restrictive than (iii); a sufficient replacement for (iii), as is easily seen, is

(iii) ${ }^{\prime}$

$$
\begin{aligned}
& G(0, t)=0, \\
& G(u, t) \rightarrow \infty \text { as } u \rightarrow \infty, \text { uniformly in } t .
\end{aligned}
$$

In addition, of course, Theorem 1 does not require that $G$ be even in $x a b$ initio.

The nonlinear Sturm-Liouville problem. The classical Sturm-Liouville problem is

$$
\begin{gathered}
{\left[p(t) x^{\prime}\right]^{\prime}+x[\lambda \rho(t)+q(t)]=0,} \\
x(0)=0, \quad x(1)=0
\end{gathered}
$$

with $p, p^{\prime}, \rho$ and $q$ continuous on $[0,1]$ and $p>0, \rho>0$ there, (20) may be transformed to the Liouville standard form

$$
x^{\prime \prime}+x[\lambda+r(t)]=0
$$

with $r(t)$ continuous (hence bounded) on [0,1]. A nonlinear relative of (22) is

$$
x^{\prime \prime}+x[\lambda+r(x, \lambda, t)]=0
$$

and it is this equation, together with (21), which is here called the "nonlinear Sturm-Liouville problem." The conditions of Theorem 1 will be satisfied by this problem if $r$ satisfies the following hypotheses:

(i) $r(x, \lambda, t)$ is defined, continuous, and bounded on $-\infty<x<\infty, \lambda<\infty$, $0 \leqq t \leqq 1$.

(ii) For fixed $\lambda$ and $t, \lambda \geqq$ some $\rho$

$$
x_{2}>x_{1} \geqq 0 \text { or } x_{2}<x_{1} \leqq \text { implies } r\left(x_{2}, \lambda, t\right) \geqq r\left(x_{1}, \lambda, t\right) \text {. }
$$

(iii) For each fixed $\lambda, r(x, \lambda, t)$ is $\operatorname{Lip}(x)$ uniformly in $t$.

This is clear except for the regenerative hypothesis, and Example 1 of $\S 1$ shows that because of (i) $[\lambda+r(x, \lambda, t)]$ is indeed regenerative. Since $r$ is bounded, $\alpha$ may be taken sufficiently negative to insure that $[\alpha+r(x, \alpha, t)]$ $\leqq 0$ for all $x$ and $t$, in which case $M$ will be zero and Theorem 1 assures the existence of solutions of $(23)+(21)$ having $i$ zeros in $(0,1)$ for $i=0,1, \cdots$. In particular, Theorem 1 applies to the linear problem $(21)+(22)$ and gives the classical result.

\section{CHAPTER II}

0. Introduction. This chapter contains results pertaining to four questions: the question of the uniqueness of the characteristic functions and numbers whose existence is assured by Theorem 1 (in $\S 1$ ), the question of the growth of the $n$th characteristic number (in \$2), the question of the "sharpness" of Theorems 1 and 2 in $\$ 3$ and finally, in $\S 4$, it is shown that the characteristic functions of a nonlinear Sturm-Liouville problem are dense in $L_{2}$ of the appropriate interval. 
1. Uniqueness of the characteristic functions and numbers. In Theorem 1 as it stands, nothing can be said about the uniqueness of either the characteristic functions or the characteristic numbers. In the first place, the hypotheses of Theorem 1 say nothing about the behavior of $F(x, \lambda, t)$ as a function of $\lambda$ when $\lambda$ is not large. Thus if $\lambda_{i}$ is a (small) characteristic number of the problem $(1)+(2)+(3)$ it may happen that for all $x$ and $t$ and some $\alpha>0$,

$$
F(x, \lambda, t)=F\left(x, \lambda_{i}, t\right), \quad \lambda_{i} \leqq \lambda \leqq \lambda+\alpha,
$$

in which case all the numbers in the interval $\left[\lambda_{i}, \lambda_{i}+\alpha\right]$ will be characteristic numbers with the same characteristic function. In addition, it may happen that there exist two distinct values of $\lambda$, say $\lambda_{1}$ and $\lambda_{2}$, and distinct functions $x_{1}\left(\lambda_{1}, t\right)$ and $x_{2}\left(\lambda_{2}, t\right)$, each satisfying $(1)+(2)+(3)$ and having the same number of zeros in $(0,1)$. An example of this type was constructed by Moore and Nehari [4] for the problem

$$
\begin{gathered}
x^{\prime \prime}+p(t) x^{2 n+1}=0, \\
x(0)=0, \quad x(1)=0
\end{gathered}
$$

$(p(t)$ continuous and positive on $[0,1])$. They were able to exhibit two distinct solutions of this problem which were nonvanishing on $(0,1)$. Since (24) is a special case of $(15)$ with $G\left(x^{2}, t\right)$ in $\operatorname{Lip}(x)$, the above problem satisfies all the hypotheses of Theorem 1.

The first of the above difficulties is easily removed by requiring that $F(x, \lambda, t)$ be strictly increasing in $\lambda$, as is automatically the case in the Nehari problem. The second difficulty is more serious. It is easily seen that the condition that $G\left(x^{2}, t\right)$ be independent of $t$ is enough to give uniqueness of the characteristic functions in the Nehari problem, but even this drastic restriction is not enough in the more general case. To see this, consider the problem

$$
\begin{gathered}
x^{\prime \prime}+x\left(\lambda^{2}+1+q\left(x^{2}\right)\right)=0, \\
x(0)=0, \quad x^{\prime}(0)=1, \\
x(1)=0,
\end{gathered}
$$

where

$$
q(s) \in C^{2} \text { and }=\left\{\begin{array}{l}
0: s \leqq 1 / \pi+1 / 2 M^{2} \\
M: s \geqq 1 / \pi+1 / M^{2}
\end{array}\right.
$$

This is a problem satisfying all the hypotheses of Theorem 1, as was shown in Chapter I, §3. The function

$$
x(t)=\frac{\sin \pi t}{\pi}
$$

is a characteristic function of this problem, having no zeros in $(0,1)$, associ- 
ated with the characteristic number $\lambda_{0}=\left(\pi^{2}-1\right)^{1 / 2}$ - because this function never penetrates the "nonlinear region" of (26). It is easily seen, however, that there is at least one other solution of $(26)+(27)+(28)$ having no zero in $(0,1)$ if $M$ is very large; loosely, for those $\lambda$ such that $\lambda^{2}<\pi^{2}-1$ the corresponding solutions of $(27)+(28)$ are initially sine functions but get "turned around" very quickly once they become large enough to penetrate the region where $q=M$.

2. Growth of the characteristic numbers. In the general problem to which Theorem 1 applies, any statement about the growth of the $n$th characteristic number $\lambda_{n}$ must necessarily involve $F$, since e.g. $\lambda, \lambda^{2}, e^{\lambda}$ are permissible $F^{\prime}$ s which give respectively $\lambda_{n}=n^{2}, \lambda_{n}=n, \lambda_{n}=2 \log n$. Nothing will be said about this problem here.

In the nonlinear Sturm-Liouville problem of Chapter I, $\$ 3$, the firstorder asymptotes of $\lambda_{n}$ and $x\left(\lambda_{n}, t\right)$ may be obtained by the usual procedure [5] by using the fact that $r(x, \lambda, t)$ in (23) satisfies a Lipschitz condition and is bounded. These results are obtained as a by-product in $\$ 4$.

3. Notes on Theorems 1 and 2. It has already been seen by an example that Theorem 1 is not "sharp" in that there exist functions $F(x, \lambda, t)$ which do not have the regenerative property but which satisfy the hypotheses of Theorem 2 (that is, functions for which the conclusions of Theorem 1 are valid but to which Theorem 1 does not apply). In fact, Theorem 2 is not sharp either. To see this, recall that the last hypothesis of Theorem 2 is used to assure that $\theta(\lambda, 1) \rightarrow \infty$ as $\lambda \rightarrow \infty$. Since by (9) $\theta(\lambda, t)$ is increasing in $t$ it is sufficient that $\theta(\lambda, b) \rightarrow \infty$ as $\lambda \rightarrow \infty$ for some $b$ in $(0,1)$. Thus if the "sups" and "infs" of the last hypothesis of Theorem 2 are taken over a subinterval $[0, b]$ instead of $[0,1]$ the theorem will still hold. Similarly it is enough for $F(x, \lambda, t)$ in Theorem 1 to have the regenerative property with respect to some subinterval $[0, b]$.

The function of Example 4, p. 448 is not regenerative on any interval $[0, b]$ unless $b=0$. The example $(\lambda \geqq 0)$

$$
x^{\prime \prime}+x\left((\lambda+2)^{-t} \log (\lambda+2)\right)=0
$$

shows, however, that the interval can not be reduced to a point, since here $F(x, \lambda, 0)=\log (\lambda+2)$ tends to $\infty$ as $\lambda$ tends to $\infty$ and thus $F$ is regenerative on the "interval" $[0,0]$. On the interval $(0,1)$, however, it is easy to see that $F(x, \lambda, t)$ is less that $1 /$ et for all $x$ and $\lambda$ and that there are no solutions of $(29)+(2)+(3)$. Thus the following remark:

REMARK. If $\check{F}(x, \lambda)$ and $\hat{F}(x, \lambda)$ are defined by

$$
\begin{aligned}
& \check{F}(x, \lambda)=\sup _{0 \leqq t \leqq 0} F(x, \lambda, t), \\
& \hat{F}(x, \lambda)=\inf _{0 \leqq t \leqq 0} F(x, \lambda, t)
\end{aligned}
$$

for some $b$ in $(0,1)$, Theorems 1 and 2 stand. Furthermore, if $F(x, \lambda, t)$ is 
not regenerative on any such interval the conclusions of Theorem 1 may not hold even though all the other hypotheses of Theorem 1 do hold.

It has already been noted that the regenerative hypothesis is the only one describing the behavior of $F(x, \lambda, t)$ as a function of $\lambda$. Thus the regenerative hypothesis is sharp for the class of $F$ 's satisfying the other hypotheses of Theorem 1, in the sense that without it the theorem may fail.

The restriction to positive $F^{\prime}$ 's in Theorems 1 and 2 gives the concavity of solution curves and is basic in the whole development given here.

4. Completeness of the characteristic functions. It is well known that the characteristic functions of a Sturm-Liouville problem on some finite interval $I$ span $L_{2}(I)$. The purpose of the present section is to show that in certain cases the characteristic functions of a nonlinear Sturm-Liouville problem have the same property. In fact, the "carry over" from the linear case to the nonlinear can be made quite striking by stating the linear results in a suitable manner. What will be proved here is that the following statement, which is true in the linear case under suitable conditions on the function $q$, remains true for the nonlinear problem, again under suitable restrictions on $q$.

Statement 1. For every non-negative integer $n$, and for every nonzero real number $p$, there exists a real number $\lambda_{p n}$ and a solution $x(p, n, t)$ of

$$
\begin{array}{r}
x^{\prime \prime}+x\left[\lambda_{p n}+q(t)\right]=0, \\
x(0)=x(1)=0,
\end{array}
$$

satisfying

(i) $x^{\prime}(p, n, 0)=p$,

(ii) $x(p, n, t)$ vanishes precisely $n$ times in $(0,1)\left({ }^{2}\right)$.

Furthermore, for each $n$ as above there exists a particular value of $p, p(n)$, for which the sequence $\{x(p(n), n, t)\}$ admits a sequence $\left\{y_{n}(t)\right\}$ such that together these sequences form a complete biorthogonal system in $L_{2}([0,1])$; every $f$ in $L_{2}([0,1])$ has the developments (converging in mean)

$$
f(t)=\sum_{n=0}^{\infty}\left(f, y_{n}\right) x(p(n), n, t)=\sum_{n=0}^{\infty}\left(f, x_{n}\right) y_{n} .
$$

That the above statement holds in the nonlinear case is the content of Theorem 3 below. Basic in the proof is the following theorem of Paley and Wiener $[6 ; 7]$ (which is as good for the present purposes as certain generalizations which have been found, e.g. [8]). The theorem is stated for any Hilbert space $H$; the one of interest here is of course real $L_{2}$.

Theorem (PAley and Wiener). Assume the sequence $\left\{f_{n}\right\}$ in $H$ differs only slightly from the complete orthonormal sequence $\left\{\phi_{n}\right\}$, in the sense that there exists a constant $\theta, 0 \leqq \theta \leqq 1$, such that

(2) For brevity a solution will be said to have the " $n$ - $p$ property" if (i) and (ii) hold. 


$$
\left\|\sum a_{n}\left(f_{n}-\phi_{n}\right)\right\|^{2} \leqq \theta^{2} \sum_{n}\left|a_{n}\right|^{2}
$$

for every finite sequence of scalars $\left\{a_{n}\right\}$. Then there exists a sequence $\left\{g_{n}\right\}$ which, with $\left\{f_{n}\right\}$, forms a complete normalized biorthogonal system; furthermore, every element of $H$ has the convergent developments

$$
f=\sum\left(f, g_{n}\right) f_{n}=\sum\left(f, f_{n}\right) g_{n} .
$$

For convenience the nonlinear Sturm-Liouville problem defined in Chapter $I, \S 3$ is restated here:

$$
x^{\prime \prime}+x[\lambda+q(x, \lambda, t)]=0,
$$

(P):

$$
x(0)=0, \quad x(1)=0 .
$$

Theorem 3. In (P), let $q(x, \lambda, t)$ be defined on

$$
\{-\infty<x<\infty, \lambda \geqq 0,0 \leqq t \leqq 1\}
$$

and satisfy there the following hypotheses:

(i) $q(x, \lambda, t)$ is continuous in $(x, \lambda, t)$.

(ii) For each fixed $\lambda$ and each positive $M$ there exists a $K(\lambda, M)$ such that whenever $\left|x_{1}\right|<M$ and $\left|x_{2}\right|<M$,

$$
\left|q\left(x_{2}, \lambda, t\right)-q\left(x_{1}, \lambda, t\right)\right| \leqq K(\lambda, M)\left|x_{2}-x_{1}\right|
$$

uniformly in $t$.

(iii) For sufficiently large $\lambda$ and each fixed $t$

$$
x_{2}>x_{1} \geqq 0 \text { or } x_{2}<x_{1} \leqq 0 \Rightarrow q\left(x_{2}, \lambda, t\right) \geqq q\left(x_{1}, \lambda, t\right) \text {. }
$$

(iv) $[\lambda+q(x, \lambda, t)] \rightarrow \infty$ as $\lambda \rightarrow \infty$ uniformly in $x$ and $t$.

(v) There exists a positive number $r$ such that $|x|^{-r} q(x, \lambda, t) \rightarrow 0$ as $|x| \rightarrow 0$ uniformly in $t$ and $\lambda$.

Then Statement 1 is true for $(\mathrm{P})$.

Example. If $G\left(x^{2}, t\right)$ satisfies Nehari's conditions and a Lipschitz condition, then $G$ satisfies all the conditions on $q(x, \lambda, t)$ in Theorem 3 . Thus the "Nehari characteristic-value problem"

$$
\begin{aligned}
x^{\prime \prime}+x G\left(x^{2}, t\right) & =-\lambda x, \\
x(0) & =x(1)=0
\end{aligned}
$$

has a set of solutions which are dense in $L_{2}([0,1])$.

Proof of Theorem 3 (begins). Hypothesis (iv) assures that $[\lambda+q(x, \lambda, t)]$ is regenerative and positive for sufficiently large $\lambda$, say $\lambda \geqq \lambda_{1}$. Let the "sufficiently large" in hypothesis (iii) be $\lambda \geqq \lambda_{2}$. One easily verifies that Theorem 2 
applies to the present problem with $\rho=\max \left[\lambda_{1}, \lambda_{2}\right]$. The end condition of interest here will be $x(0)=x(1)=0, x^{\prime}(0)=p$. The $\alpha$ of Theorem 2 may be taken as any non-negative number; the point of the following lemma, however, is that a positive $\alpha$ may be chosen such that the corresponding value of $M$ (the least number of zeros in $(0,1)$ of any solution of $(30)$ when $\lambda=\alpha$ ) is zero.

LEMMA 8. There exists a positive number $\alpha$ such that if $|p|$ is sufficiently small the solution of (30) for $\lambda=\alpha$ which satisfies the initial conditions $x(0)=0$, $x^{\prime}(0)=p$, does not vanish in $(0,1)$.

Proof. Take $\alpha=1 / 2$. It is possible to find a $u>0$ such that

$$
|x| \leqq u \Rightarrow \sup _{t}|q(x, 1 / 2, t)| \leqq 1 / 4 \text {. }
$$

This is a consequence of hypothesis (v) on $q$. Let $|p|$ be less than $u$ (this is the precise definition of "sufficiently small" in the statement of the lemma). Let $x(t)$ be the solution of

$$
\begin{gathered}
x^{\prime \prime}+x[1 / 2+q(x, 1 / 2, t)]=0, \\
x(0)=0, \quad x^{\prime}(0)=p
\end{gathered}
$$

for some $p$ restricted as above. As long as $|x| \leqq u,[1 / 2+q(x, 1 / 2, t)] \geqq 1 / 4$ $>0$, and so the graph of $x(t)$ is concave toward the $t$ axis. As long as the graph is concave, however, $x(t)$ can not exceed $p t$, which is less than $u$. Thus $|x(t)| \leqq u$ until $t_{1}$, the first zero of $x(t)$ in $(0,1)$, and on $\left[0, t_{1}\right][1 / 2+q(x, 1 / 2, t)]$ $<1 / 2+1 / 4=3 / 4$. Defining $\theta(t)$ as in (7), (9) becomes

$$
\theta^{\prime}=1+\sin ^{2} \theta[1 / 2+q(x, 1 / 2, t)-1],
$$

hence $\theta^{\prime}<1$ on $\left[0, t_{1}\right]$ and $\theta\left(t_{1}\right)<t_{1}<\pi$. This is a contradiction, since $x\left(t_{1}\right)$ $=0 \Rightarrow \theta\left(t_{1}\right)=\pi$. Thus $t_{1}$ does not exist, i.e. $x(t)$ has no zero in $(0,1)$. I

In the course of the following proof $|p|$ will be restricted frequently. It is here stipulated that $|p|$ will always be taken so small that Lemma 8 applies. It follows then that $\alpha$ of Theorem 2 may be taken as $1 / 2$ and $M$ as zero, and thus (for small $|p|$ ) Statement 1 up to the line beginning "Furthermore ..." is true.

It also follows that all characteristic numbers which arise may be assumed to be bounded from below by a positive number, namely $1 / 2$.

In the sequel it is not assumed that $x(n, p, t)$ and $\lambda_{p n}$ are uniquely determined by $n$ and $p$, since for any given $p$ and $n$ Theorem 2 merely assures the existence of at least one number $\lambda_{p n}$ leading to a solution $x(n, p, t)$ enjoying the $n$ - $p$ property. Once $\lambda_{p n}$ is selected, however, the corresponding function $x(n, p, t)$ is uniquely determined (because by hypothesis (ii) solutions are uniquely determined by their initial values).

The next lemma provides an upper bound on $\lambda_{p n}$ in terms of $n$ : 
Lemma 9. There exists an $M(n)$ such that $\lambda_{p n}<M(n)$ uniformly in $p$.

Proof. Take $M(n)$ so large that $M(n)+q(x, M(n), t) \geqq(n+2)^{2} \pi^{2}$ for all $x$ and $t$. This is possible because by hypothesis (iv) $[\lambda+q(x, \lambda, t)] \rightarrow \infty$ as $\lambda \rightarrow \infty$. Let $p$ and $n$ be fixed and suppose $b^{2}$ is a $\lambda_{p n}$ such that $b^{2}>M(n)$. Let $x(t)$ be the corresponding solution with the $n$-p property and define $\theta(t)$ as in (7). In the present instance (9) becomes

$$
\theta^{\prime}=1+\sin ^{2} \theta\left[b^{2}+q\left(x, b^{2}, t\right)-1\right] .
$$

Let $\phi(t)$ be the unique solution of

$$
\phi^{\prime}=1+\sin ^{2} \phi\left[(n+2)^{2} \pi^{2}-1\right]
$$

which vanishes at $t=0$. Let $C=(n+2) \pi$. Subtracting (35) from (34)

$$
\begin{aligned}
(\theta-\phi)^{\prime} & =\left(\sin ^{2} \theta-\sin ^{2} \phi\right)\left(C^{2}-1\right)+\sin ^{2} \theta\left[b^{2}-c^{2}+q\left(x, b^{2}, t\right)\right] \\
& =\left(\frac{\sin \theta-\sin \phi}{\theta-\phi}\right)(\sin \theta+\sin \phi)\left(C^{2}-1\right)(\theta-\phi)+h,
\end{aligned}
$$

where $h \geqq 0$. Let the first term in (36) be written $f(t)(\theta-\phi)$. Since $\theta$ and $\phi$ are continuous in $t$, so is $f$ (clearly there is no difficulty where $\theta=\phi$ ). Thus, using an integrating factor $\exp \left(-\int^{t} f(s) d s\right)$

$$
\left[\exp \left(-\int^{t} f(s) d s\right)(\theta-\phi)\right]^{\prime}=\exp \left(-\int^{t} f(s) d s\right) h(t) \geqq 0
$$

or, since $(\theta-\phi)(0)=0$,

$$
(\theta-\phi)(t) \geqq 0 \text {. }
$$

(35) is easily solved, however, to give $\phi(1)=(n+2) \pi$, when by (37) $\theta(1)$ $\geqq(n+2) \pi$. This is a contradiction, because since $x(t)$ has $n$ zeros in $(0,1)$, necessarily $\theta(1)=(n+1) \pi$. I

LEMMA 10. For every non-negative integer $n$ there exists $a p(n)$ and a characteristic number $\lambda_{p(n), n}$ of $(\mathrm{P})$ such that if $x(t)$ is the corresponding solution of (P) with the $n-p$ property (i.e. $x(t)=x(p(n), n, t)$ ), the following bounds hold (uniformly in $t$ in 1 and 3):

1.

$$
|x(t)|<\frac{2|p(n)|}{\lambda_{p(n), n}}
$$

2.

$$
\frac{2|p(n)|}{\lambda_{p(n), n}}<\min \left\{1,\left(\frac{\lambda_{p(n), n}}{12(n+1)^{3} \pi^{2}}\right)^{1 / r}\right\}
$$

3. $\quad\left|q\left(x(t), \lambda_{p(n), n}, t\right)\right|<\frac{\lambda_{p(n), n}}{2}|x(t)|$.

Here $r$ is the $r$ of hypothesis (v). 
Proof. 1. Let $n$ be fixed and choose a $p$ (which will depend on $n$ ) and a suitable characteristic number $\lambda_{p, n}$ (write $b^{2}$ for convenience) such that uniformly in $|x| \leqq(2 / b)|p|$

$$
\left|q\left(x, b^{2}, t\right)\right|<\frac{|x| r}{2} b .
$$

That this choice is possible follows from the preceding lemmas. In the first place, by Lemma 9 there is an $M$ (which depends on $n$ but here $n$ is fixed) such that $b^{2} \leqq M$, and by Lemma $8 b^{2}>1 / 4$, hence

$$
\frac{2}{|x|^{r} b}\left|q\left(x, b^{2}, t\right)\right| \leqq \frac{2}{|x|^{r}(1 / 2)} \sup _{\lambda \leqq M} \check{q}(x, \lambda) .
$$

Letting the right side of this inequality be $g(x)$, it follows from the continuity of $q$ and hypothesis (v) that $g(x)$ is continuous and tends to zero as $x$ tends to 0 . Thus if $|p / b|$ is sufficiently small, that is if $|x|$ is restricted to lie near zero, then (38) must hold. By Lemma $8|p / b| \rightarrow 0$ as $|p| \rightarrow 0$, so the indicated choice is possible.

2. Decrease $|p|$ if necessary so that $|p| / b<1 / 2$ and

$$
\frac{|p|}{b^{1+1 / r}}<\frac{1}{2}\left(\frac{1}{12(n+1)^{3} \pi^{2}}\right)^{1 / r}
$$

Again, Lemma 9 assures that this is possible. Thus it has been shown that $p(n)$ can be chosen so that the second and third bounds of the present lemma hold whenever the first one does.

3. With any $p$ subject to the above, and the corresponding $b$, define successive approximants to the associated characteristic function $x(t)$ by

$$
\begin{aligned}
x_{0}(t) & =p \frac{\sin b t}{b}, \\
x_{1}(t) & =p \frac{\sin b t}{b}-\int_{0}^{t} \frac{\sin b(t-s)}{b} x_{0}(s) q\left(x_{0}(s), b^{2}, s\right) d s, \\
\text {. . . . . . . . . . . . . . . . . . . . } & \text {. } x_{j}(s) q\left(x_{j}(s), b^{2}, s\right) d s . \\
x_{j+1}(t) & =p \frac{\sin b t}{b}-\int_{0}^{b}(t-s)
\end{aligned}
$$

4. Clearly $\left|x_{0}(t)\right|<2|p / b|$. Assume inductively that

$$
\left|x_{j}(t)\right|<\frac{2|p|}{b} \text {. }
$$

Then, using the results of steps 1 and 2 in (39) 


$$
\begin{aligned}
\left|x_{j+1}(t)\right| & <\frac{|p|}{b}+\frac{1}{b} \frac{2|p|}{b} \frac{b}{2} \sup _{i}\left|x_{j}(t)\right|^{r} \\
& <\frac{|p|}{b}\left[1+1^{r}\right] \leqq \frac{2|p|}{b}
\end{aligned}
$$

and by induction (40) holds for all $j$.

5. From (39) and what has already been done, there is a $K$-independent of $j, t_{2}$ and $t_{1}$-such that

$$
\left|x_{j+1}\left(t_{2}\right)-x_{j+1}\left(t_{1}\right)\right| \leqq K\left|t_{2}-t_{1}\right| \text {. }
$$

To see this, assume $t_{1}>t_{2}$ and write

$$
\begin{aligned}
x_{j+1}\left(t_{1}\right)-x_{j+1}\left(t_{2}\right)= & \int_{0}^{t_{2}}\left[\frac{\sin b\left(t_{1}-s\right)}{b}-\frac{\sin b\left(t_{2}-s\right)}{b}\right] x_{j}(s) q d s \\
& +\int_{t_{2}}^{t_{1}} \frac{\sin \left(t_{1}-s\right)}{b} x_{j}(s) q\left(x_{j}(s), b^{2}, s\right) d s, \\
= & \left(t_{2}-t_{1}\right) \int_{0}^{t_{2}} \cos b(\xi-s) x_{j}(s) q d s \\
& +\int_{t_{2}}^{t_{1}} \frac{\sin b\left(t_{1}-s\right)}{b} x_{j}(s) q\left(x_{j}(s), b^{2}, s\right) d s .
\end{aligned}
$$

Then note that both integrands are bounded.

6. By step 5 and $(40)$ the sequence $\left\{x_{j}(t)\right\}$ has a uniformly convergent subsequence, and the limit function $y(t)$ must satisfy (40) and also, by (39), the integral equation

$$
y(t)=p \frac{\sin b t}{b}-\int_{0}^{t} \frac{\sin b(t-s)}{b} y(s) q\left(y(s), b^{2}, s\right) d s .
$$

By differentiating (41) it is seen that $y(t)$ is a solution of (30). Also, $y(0)=0$ and $y^{\prime}(0)=p$, hence by uniqueness $y(t)=x(t)$ and $x(t)$ satisfies $(40)$.

LEMMA 11. For every non-negative integer $n$ there exists a solution $x(t)$ of (P) having $n$ zeros in $(0,1)$ and such that for some constant $u$

$$
\sup _{t}\left|u x(t)-\frac{\sin (n+1) \pi t}{(n+1) \pi}\right|<\frac{1}{(n+1)^{2} \pi^{2}} .
$$

Proof. Choose a $p(n)$ (write $p$ ) and a $\lambda_{p(n), n}$ (write $b^{2}$ ) such that for the corresponding $n-p$ solution $x(t)$ of $(\mathrm{P})$ the bounds of Lemma 10 hold. This is possible by Lemma 10. It will be shown that this $x(t)$ is the $x$ of the lemma with $u=1 / p$. First, it is easy to see that 


$$
x(t)=p \frac{\sin b t}{b}-\int_{0}^{t} \frac{\sin b(t-s)}{b} x(s) q\left(x(s), b^{2}, s\right) d s,
$$

hence

$$
\begin{aligned}
\left|\frac{1}{p} x(t)-\frac{\sin b t}{b}\right| & \leqq \frac{1}{|p|} \int_{0}^{t}\left|\frac{\sin b(t-s)}{b} x(s) q\left(x(s), b^{2}, s\right)\right| d s \\
& \leqq \frac{1}{|p|} \frac{1}{b} \frac{2|p|}{b} \frac{b}{2}\left(\frac{2|p|}{b}\right)^{r} \\
& \leqq \frac{1}{12(n+1)^{3} \pi^{2}}
\end{aligned}
$$

Thus the proof will be complete if it is shown that

$$
\left|\frac{\sin b t}{b}-\frac{\sin (n+1) \pi t}{(n+1) \pi}\right|<\frac{1}{2(n+1)^{2} \pi^{2}} .
$$

By (43) and the fact that $x(1)=0, b$ must be "close to" $m \pi$ for some integer $m$. Since for the $x(t)$ of interest here $|q(x, \lambda, t)|<1$, two applications of an argument as in Lemma 9 will yield that $n<m<(n+2)$. Defining $\delta$, then, by $b \equiv[(n+1) \pi+\delta]$ it is known that $-\pi<\delta<\pi$.

An elementary estimate shows that (44) will hold if

$$
|\delta|<\frac{1}{6(n+1)^{2} \pi^{2}} .
$$

By (43) at $t=1$, however,

$$
\frac{\sin |\delta|}{(n+2) \pi}<\frac{\sin |\delta|}{(n+1) \pi+\delta}=\left|\frac{\sin b}{b}\right| \leqq \frac{1}{12(n+1)^{3} \pi^{2}}
$$

or

$$
\sin |\delta|<\frac{n+2}{12(n+1)^{3} \pi^{2}}
$$

or

$$
|\delta|<\frac{1}{6(n+1)^{2} \pi^{2}} \cdot \mid
$$

Proof of Theorem 3 (ends). For each $n=0,1,2, \cdots$ let $x_{n}(t)$ be a solution of $(\mathrm{P})$ having $n$ zeros in $(0,1)$ and satisfying $(42)$ with $u=u_{n}$. If $a_{0}, a_{1}, \cdots, a_{m}$ are any real numbers 


$$
\begin{aligned}
& \left(\sum_{j=0}^{m} a_{j}\left[u_{j} x_{j}-\frac{\sin (j+1) \pi t}{(j+1) \pi}\right]\right)^{2} \\
& \quad \leqq\left(\sum_{j=0}^{m} a_{j}^{2}\right)\left(\sum_{j=0}^{m}\left[u_{j} x_{j}-\frac{\sin (j+1) \pi t}{(j+1) \pi}\right]^{2}\right) \\
& \quad \leqq \sum_{j=0}^{m} a_{j}^{2} \sum_{j=0}^{m} \frac{1}{(j+1)^{4} \pi^{4}}<\sum_{j=0}^{m} a_{j}^{2} \sum_{j=0}^{\infty} \frac{1}{(j+1)^{4} \pi^{4}} \\
& \quad<\frac{1}{2} \sum_{j=0}^{m} a_{.}^{2}
\end{aligned}
$$

Thus, integrating from zero to one

$$
\left\|\sum_{j=0}^{m} a_{j}\left[u_{j} x_{j}-\frac{\sin (j+1) \pi t}{(j+1) \pi}\right]\right\|^{2}<\frac{1}{2} \sum_{j=0}^{m} a_{j}^{2} .
$$

By the Paley and Wiener Theorem, and the fact that the sequence

$$
\left\{\frac{\sin (j+1) \pi t}{(j+1) \pi}\right\}
$$

is complete and orthogonal in $L_{2}([0,1])$, the sequence $\left\{u_{j} x_{j}(t)\right\}$ is dense in $L_{2}([0,1])$ and admits a sequence $\left\{\phi_{j}\right\}$ such that together these are a complete biorthogonal system. Clearly, then, the two sequences $\left\{x_{j}(t)\right\},\left\{u_{j} \phi_{j}\right\}$ also form a complete biorthogonal system and the theorem follows. I

\section{References}

1. Zeev Nehari, Characteristic values associated with a class of nonlinear second-order differential equations, Mathematics Division Air Force Office of Scientific Research Report No. AFOSR TN 59-1262.

2. Earl A. Coddington and Norman Levinson, Theory of ordinary differential equations, McGraw-Hill, New York, 1955, Chapter 2, Theorem 4.1.

3. Zeev Nehari, On a class of nonlinear second-order differential equations, Trans. Amer. Math. Soc. 95 (1960), 101-123.

4. R. A. Moore and Zeev Nehari, Nonoscillation theorems for a class of nonlinear differential equations, Trans. Amer. Math. Soc. 93 (1959), 30-52.

5. Earl A. Coddington and Norman Levinson, op. cit., p. 184.

6. R. E. A. C. Paley and N. Wiener, Fourier transforms in the complex domain, Amer. Math. Soc. Colloq. Publ. Vol. 19, New York, 1934, p. 100.

7. Frigyes Riesz and Bela Sz.-Nagy, Functional analysis, Frederick Ungar Publishing Co., New York, 1955, §86. 975-978.

8. Bela Sz.-Nagy, Expansion theorems of Paley-Wiener type, Duke Math. J. 14 (1947),

Massachusetts Institute of Technolugy,

Cambridge, Massachusetts 